\title{
Novel Epstein-Barr virus immunoglobulin G-based approach for the specific detection of nasopharyngeal carcinoma.
}

\begin{abstract}
Purpose This study was designed to find a reliable Epstein-Barr virus (EBV) immunoglobulin (Ig) G-based diagnostic/screening test for nasopharyngeal carcinoma (NPC) able to demarcate between the NPC-related seropositivity of EBV IgG antibodies and that of other head and neck cancer (HNCA) and control groups. The NPC-associated immunosuppression affects EBV IgA much more than IgG, leading to inconsistent detection of NPC using EBV IgA antibodies. Materials and methods One hundred twenty-two HNCA patients, 42 NPC, 66 laryngeal carcinoma, and 14 hypopharyngeal carcinoma and 3 groups of 100 control subjects were enrolled in this study. Enzyme-linked immunosorbent assay (ELISA) was used to find a specific cutoff value for the NPC-related seropositivity of EBV IgG antibodies. Results NPC group showed higher serum level of EBV IgG antibodies than control and other HNCA groups $(\mathrm{P}<.05)$. However, the traditional cutoff value, mean +2 SDs of control subjects, failed to demarcate the seropositives of NPC patients from those of healthy population $(\mathrm{P}>$ $.05)$. The new cutoff value, mean +2 SDs of the seropositives group of control subjects who had already been grouped by the traditional cutoff value, proved successful. It succeeded to demarcate between the NPC-related EBV IgG seropositivity and that issued from the persistent, latent, or reactivated $\mathrm{EBV}$ infection in the population $(\mathrm{P}<.05)$. The sensitivity/specificity of NPC detection by the new cutoff-based ELISA kit, $76.19 \%$ and $86 \%$, was close or higher than that of EBV IgA antibodies. Conclusion EBV IgG-based ELISA could be used for the diagnosis of NPC using a new cutoff threshold that excludes the population baseline of EBV IgG seropositivity.
\end{abstract}

Keyword: Novel Epstein-Barr virus; Immunoglobulin G-based. 